\section{INTEGRACIÓN DE CONOCIMIENTOS PARA REDUCIR RIESGOS POR DERRAMES DE HIDROCARBUROS EN FUENTES HÍDRICAS}

Verónica Zapata Gómez ${ }^{1 *}$, Manuela Cortés Cano ${ }^{1}$, Olga Lucía Amaya Moncada ${ }^{1}$, Laura Osorio Cano ${ }^{1}$ y Edna Margarita Rodríguez Gaviria ${ }^{1}$

\section{RESUMEN}

El corregimiento de San Cristóbal, ubicado en la periferia occidental de la ciudad de MedellínColombia, se ha visto expuesto a diversos factores de riesgo desde principios del siglo XXI, debido a la contaminación con hidrocarburos de sus fuentes hídricas. Esto contribuye al aumento de la vulnerabilidad, a causa de la desinformación; la ausencia de un plan de gestión del riesgo y de entes gubernamentales y privados en los Comités de Gestión del Riesgo de Desastres. La metodología utilizada integra: la recopilación de información por medio de bases de datos, noticias, elaboración de encuestas y ejercicios participativos; la identificación de actores; el análisis de causas que generan amenaza a la prestación del servicio de agua potable; un análisis territorial; la elaboración de cartografía; y la implementación de medidas educativas. La aplicación de la metodología ha permitido plantear una discusión sobre las condiciones de vulnerabilidad generadas por la desarticulación con la planificación del desarrollo local y por los problemas de vigilancia y asesoramiento a la comunidad, además de proponer medidas correctivas y prospectivas para la reducción del riesgo.

\section{PALABRAS CLAVES}

Agua, Riesgo, Reducción, Vulnerabilidad, Hidrocarburos, Colombia

\section{INTEGRATION OF KNOWLEDGE TO REDUCE RISKS FROM HYDROCARBON SPILLS IN WATER SOURCES}

\begin{abstract}
Located on the western outskirts of Medellin, San Cristobal Village has been exposed to a variety of risk factors, since early 21st century, due to hydrocarbons contamination of its water resources. This, contributes to an increase of the vulnerability, caused by disinformation; lack of a risk management plan, and governmental and private entities in the risk management committees. The applied methodology integrates: a compilation of information throughout databases, news, surveys, and participative workshops; participants identification; analysis to causes which are hazardous to the potable water service; territorial analysis; cartography elaboration analysis; and, educative measures implementation. The application of this methodology has allowed for, raise a discussion about the conditions of vulnerability generated by the disarticulation with local development planning, and by the surveillance and advisory of the community problems, besides the proposal of prospective and corrective measures for risk reduction.
\end{abstract}

\section{KEYWORDS}

Water, Risk, Reduction, Vulnerability, Hydrocarbons, Colombia

\author{
1. Facultad de Arquitectura \\ e Ingeniería, Institución \\ Universitaria Colegio Mayor \\ de Antioquia, Medellín, \\ Colombia. \\ *Autor de correspondencia: \\ verozapatao713@gmail.com \\ DOI: \\ https://doi.org/10.55467/ \\ reder.v6i1.9o
}

\section{RECIBIDO}

25 de enero de 2021

\section{ACEPTADO}

10 de septiembre de 2021

\section{PUBLICADO}

1 de enero de 2022

\section{Formato cita}

Recomendada (APA):

Zapata Gómez, V., Cortés Cano, M., Amaya Moncada, O.L., Osorio Cano, L. \& Rodríguez Gaviria, E.M. (2022). Integración de conocimientos para reducir riesgos por derrames de hidrocarburos en fuentes hídricas. Revista de Estudios Latinoamericanos sobre Reducción del Riesgo de Desastres REDER, 6(1), 8398. https://doi.org/10.55467/ reder.v6i1.90

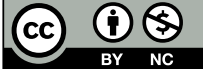

Todos los artículos publicados en REDER siguen una política de Acceso Abierto y se respaldan en una Licencia CreativeCommons Atribución-NoComercial 4.0 Internacional.

Revista de Estudios Latinoamericanos sobre Reducción del Riesgo de Desastres (REDER)

Diseño: Lupe Bezzina 


\section{INTRODUCCIÓN}

La contaminación de hidrocarburos produce un cambio en las características organolépticas del agua que produce problemas a la salud de los consumidores, igualmente a ecosistemas que pueden sufrir afectaciones por contaminación. La contaminación puede presentarse de dos formas generales: puntuales y sistemáticas. Las primeras ocurren en los cuerpos de agua donde generalmente no hay presencia de hidrocarburos. Las segundas son habituales y se caracterizan por la presencia de aguas contaminadas por la actividad antrópica que se realiza en ellas (Prieto Diaz \& Martínez de Villa Pérez, 1999). Por otro lado, el daño ambiental causado por derrames de hidrocarburos incrementa los riesgos de sobrepasar los límites de la sostenibilidad de las empresas dedicadas a la explotación de recursos agotables como el carbón o el petróleo (Amaya Navas, 2012; Gudynas, 2003). En México, Maldonado-Chávez et al. (2010) reportan cómo la industria petrolera es una fuente importante de contaminación del suelo: las actividades de perforación, extracción, conducción y transformación del petróleo en zonas petroleras han originado la contaminación del suelo y el agua ocasionada por derrames, fugas, filtraciones, lodos y recortes de perforación desde hace más de 40 años. En Colombia, el sector petrolero ha tenido un crecimiento en la economía a partir del siglo XXl; este sector es estratégico para la economía por su alta participación en el producto interno bruto (Cuartas Ñ́stez et al., 2014). Sin embargo, la contaminación por este tipo de sustancias es cada vez mayor, afectando la flora, fauna e incluso la salud de los seres humanos.

En Colombia, se han presentado derrames por diversas circunstancias: voluntaria, accidental o por atentados de grupos al margen de la ley, entre otras. "En algunas circunstancias, dichas situaciones se han generado en sectores del país de difícil acceso, impidiendo el desarrollo de estrategias de vigilancia y control o en el caso dado de recuperación, por ejemplo los derrames causados por grupos al margen de la ley desde hace 30 años, lo que ha generado una alteración en la calidad del paisaje y en los servicios ecosistémicos, que proporcionan el hábitat para el normal desarrollo de la vida de los seres humanos; por esto es necesario enfatizar que la problemática no es solo ambiental, puesto que la contaminación de la naturaleza ya sea en suelos o aguas, conlleva a generar grandes impactos económicos en la sociedad, en donde las actividades como la pesca, consumo de agua, o el cultivo de especies primordiales son las primeras afectadas, desencadenando un impacto social considerable, donde la población se ve afectada directa 0 indirectamente" (Velásquez Arias, 2017).

En Colombia, hasta noviembre de 1998 se presentaron 920 ataques contra la infraestructura petrolera, 575 de ellos en el oleoducto Caño Limón-Coveñas. Dichos ataques han perjudicado ecosistemas, fuentes de producción y abastecimiento de agua de las comunidades aledañas al oleoducto; además, por estos ataques se pueden presentar fallas técnicas en la infraestructura que genera problemas en las líneas de transporte de hidrocarburos que a su vez generan derrames. Las áreas perjudicadas por los derrames de petróleo se ubican principalmente en la zona alta de la llanura araucana, en la región de la cuenca del río Catatumbo; departamentos de Santander, Cesar y Sucre, principalmente, y en los departamentos del Putumayo y Nariño (Benavides López De Mesa et al., 2005).

La contaminación por hidrocarburos se ha experimentado también en el Corregimiento de San Cristóbal - Medellín (Departamento de Antioquia, Colombia), donde las fuentes de abastecimiento de agua se han visto afectadas en varias oportunidades por contaminación de hidrocarburos, dejando sin suministro a las viviendas que se abastecen de la planta potabilizadora del corregimiento administrada por Empresas Públicas de Medellín (EPM). En el año 2011 (El Colombiano, 2011) se registró un derrame de hidrocarburos en la zona rural de la vereda Yolombo por un derrumbe que desplazó la tubería, ocasionando daño en el poliducto del corregimiento por la susceptibilidad que tiene la zona a movimientos en masa de magnitud alta y media como producto de la combinación de factores naturales y antrópicos (Alcaldía de Medellín, 2015).

Posteriormente, se dio a conocer la noticia de la suspensión en el suministro de agua en el corregimiento, por el vertimiento de hidrocarburos en dos quebradas que surten el acueducto. Después de una investigación hecha por la Corporación Autónoma Regional del Centro de Antioquia (Corantioquia) "se presume que la responsable de dicho vertimiento podría ser la Concesión Desarrollo Vial al Mar S.A.S-Devimar, quien construye la segunda fase del Túnel de Occidente" (Caracol Radio Medellín, 2019). 
El impacto de los macroproyectos es la principal problemática ambiental del Corregimiento, ya que contribuyen al deterioro ambiental, la disponibilidad del agua que principalmente se usa para el riego de cultivos agrícolas, la contaminación de los nacimientos y quebradas por aguas residuales y mal manejo de los residuos sólidos, el crecimiento acelerado y desordenado de la construcción informal y, en general, el manejo inadecuado de los recursos naturales. Estos factores ponen en peligro la sostenibilidad ambiental y la riqueza natural e impactan de manera directa la calidad de vida de los pobladores del Corregimiento (Alcaldía de Medellín, 2015).

La Gestión del Riesgo de Desastres en Colombia es regida por la Ley 1523 de 2012 por la Cual se Adopta la Política Nacional de Gestión del Riesgo (Congreso de la República de Colombia, 2012), por la cual fueron derogados los principales sustentos legales del Sistema Nacional de Prevención y Atención de Desastres (SNPAD), para dar paso a la creación del Sistema Nacional de Gestión del Riesgo de Desastres (SNGRD) y la adopción de una política pública en la materia. La ley define la gestión del riesgo como un proceso social orientado a la formulación, ejecución, seguimiento, evaluación de proyectos, y para tomar las medidas pertinentes para la reducción del riesgo y el manejo de desastres, con el propósito de contribuir a la seguridad, el bienestar, la calidad de vida de las personas y al desarrollo sostenible.

En Suramérica son recurrentes los desastres asociados a fenómenos naturales, socio naturales y causados por la actividad humana, tales como sismos, inundaciones, deslizamientos o contaminación del recurso hídrico (Galeano Balvuena, 2015). Estos desastres afectan el desarrollo de los diferentes territorios impactados, impiden y retrasan la obtención de las metas de bienestar social trazadas por los gobiernos y las acciones comunales de los sectores específicos.

Para evitar estos impactos se debe realizar una adecuada gestión del riesgo, implementando estrategias eficientes de prevención, atención y recuperación de desastres las cuales traen su sustento en el cumplimiento del Marco de Sendai, en los artículos 38 y 42 de la Política Nacional de Gestión del Riesgo, propendiendo por el cumplimiento especialmente del objetivo de Desarrollo Sostenible $N^{0} 6$ denominado Agua Limpia y Saneamiento (Asamblea General de Las Naciones Unidas, 2015).

En consecuencia, los desastres no son naturales, si no por el contrario, son la resultante de un proceso de construcción de condiciones de vulnerabilidad causados por el hombre y de un desarrollo inadecuado e insostenible en el tiempo (Mesa de Concertación para la Lucha contra la Pobreza \& Cáritas del Perú, 2009).

Con base en lo anterior, es importante tener en cuenta ayudas tecnológicas como los "sistemas de información geográfica para tener información clara acerca de las zonas afectadas por la contaminación del recurso hídrico y poder realizar planes de acción a la comunidad vulnerable" (Gil Castrillón, 2018).

Con este fin, se plantearon entonces medidas de reducción mediante acciones participativas a partir del desarrollo de una propuesta de integración del conocimiento local del riesgo por derrame de hidrocarburos en el Corregimiento, la cual consiste en la búsqueda de datos históricos acerca de la problemática ya mencionada, mediante información que pueda proporcionar noticias y bases de datos para tomar acciones frente a los actores de riesgo y así "categorizar por eventos de desastres históricos para detectar los vacíos o elementos críticos de las empresas prestadoras del servicio público de acueducto que deben suplir para cumplir con los requisitos y lineamientos establecidos desde la normatividad buscando contribuir a la seguridad, el bienestar, la calidad de vida de las personas y al desarrollo sostenible" (Gil Castrillón, 2018).

\section{METODOLOGÍA}

\section{Técnicas analíticas}

En la ejecución de esta investigación se utilizaron técnicas analíticas de elaboración de mapas conceptuales, matrices, líneas de tiempo y matrices para la sistematización de información proveniente de fuentes gubernamentales y privadas en base a Planes de Desarrollo Municipal actuales y anteriores, Planes de Desarrollo Departamental, Planes de Ordenamiento Territorial Municipal, Planes de Gestión del Riesgo Municipal, mapas y bases de datos catastrales, mapas con cartografía básica que contiene datos espaciales topográficos, datos espaciales administrativos, datos espaciales de infraestructura, institucionales y datos espaciales geográficos y, por último, en imágenes como ortofotos (Gil Castrillón, 2018; Rodríguez-Gaviria, 2016). 
El plan de captura de información de este tipo también es de utilidad para tener relaciones entre los diferentes actores implicados en la investigación, para el cumplimiento de los objetivos a través del uso de métodos de investigación de acción participativa, que van desde entrevistas, realización de talleres y elaboración de transectos con líderes comunitarios y habitantes de la zona, entre otros (Williams et al., 2018).

\section{Descripción metodológica}

\section{Análisis de fuentes de amenaza}

a. Análisis de la ocurrencia de desastres en el área de estudio mediante la recopilación de información proveniente de noticias, bases de datos de desastres, información localizada en instituciones del Sistema Nacional de Gestión del Riesgo local (Medellín-Antioquia).

b. Determinación del área de estudio a través de la información recolectada en el paso anterior y el uso de software para cartografía como el ArcGIS, bien sea por la frecuencia de ocurrencia por medio de una línea de tiempo, por la gravedad de los impactos (base de datos, noticias) o por la cantidad de personas impactadas (noticias, revisión de bibliografía) o por la cantidad de infraestructura afectada; por medio de fuentes de información secundaria. Se busca identificar el tamaño y la dispersión de las zonas afectadas y su población.

c. Identificación de las fuentes que generan amenaza a la prestación del servicio de agua potable en la zona, dándose a conocer todas las fuentes posibles de amenaza por medio de un mapa conceptual. Esta información se obtiene a través de la búsqueda de noticias.

\section{Mapa de actores}

a. Construcción de mapa de actores para identificar y visualizar interrelaciones entre ellos en la gestión del riesgo y tomadores de decisiones involucrados. Se busca identificar las acciones y responsabilidades en la gestión del riesgo. Cómo se dan los procesos de coordinación, cooperación y traspaso de información a través de los grupos y en dónde se presentan potenciales conflictos.

Esta construcción se lleva a cabo por medio de la revisión de información contenida en el Plan de Desarrollo, como en el Plan de Ordenamiento Territorial del Municipio de Medellín, además de otros estudios e informes y en el desarrollo de entrevistas y recorridos de campo.

A través del mapa de actores es posible observar los diferentes grupos relacionados con la gestión del riesgo de acuerdo con su presencia territorial correspondiente a las escalas nacional, regional y local y una clasificación por niveles: primer nivel, aquellos que producen o centralizan la información (incluyendo políticas) y la transmiten a otras partes; de segundo nivel, los principales interesados en la gestión de emergencias, y por lo general, obtienen la información y las instrucciones de las instituciones de primer nivel. Desempeñan una función clave debido a sus diferentes funciones, conocimientos y capacidades técnicas. Un tercer nivel, que son aquellas instituciones públicas que desempeñan un papel importante en la planificación estratégica, tal como los del primer nivel y actúan como un puente para la comunicación de la gestión del riesgo entre las autoridades locales y el público en general. En el cuarto nivel, miembros de la comunidad, organizaciones comunitarias, instituciones públicas y privadas, los cuales son propensos a ser afectados y que se convierten en los principales beneficiarios de una adecuada gestión del riesgo. Además, se incluyeron las funciones que cumplen cada uno de estos en el corregimiento de San Cristóbal.

b. Estos grupos desempeñan un papel relevante como multiplicadores de las medidas de prevención y concienciación del riesgo de contaminación por hidrocarburos en su comunidad.

También era necesario identificar el flujo de información que existe entre los actores para mostrar los diferentes tipos de interacciones que tienen lugar entre las partes interesadas. Se consideraron tipos de interacciones como el flujo de información, la 
cooperación y los potenciales conflictos. Al identificar los actores se buscó recopilar la mayor cantidad de información sobre las condiciones de riesgo asociadas a la contaminación por hidrocarburos mediante un enfoque global de los actores, abarcando la mayor cantidad de actores, sus relaciones y la incorporación de factores sociales, económicos, ambientales. Se recurrió al uso de herramientas de captura de información como entrevistas, encuestas y visitas de campo.

\section{Cartografiando afectaciones}

a. Mediante un análisis territorial, se plantearon ejercicios participativos de construcción de cartografía para localizar los elementos expuestos en la zona de estudio elegida. Los instrumentos fueron mapeo comunitario por medio de un formato de entrevista virtual dirigido a la comunidad para la delimitación de la zona con habitantes afectados.

\section{Formulación de estrategias}

a. Indagar con la Junta de Acción Local del Corregimiento en la reducción del riesgo (protocolos, planes de gestión del riesgo, planes de contingencia, y otros instrumentos de planificación), qué poseen y qué pueden articular a los ejercicios de planificación estratégica del corregimiento de San Cristóbal, para que desde lo social, económico y ambiental pueda lograrse acciones en el corto, mediano y largo plazo.

b. Se planean e implementan estrategias de educación y participación para la gestión del riesgo de contaminación por hidrocarburos por medio de capacitaciones (videos educativos y de sensibilización, debates, por medio de alternativas virtuales, llegar por medio de plataforma Facebook, Periódico, etc.).

\section{RESULTADOS Y ANÁLISIS}

\section{Análisis de fuentes de amenaza}

Para el logro de este objetivo se realizó una matriz de hechos históricos (ver Tabla 1) con el fin de recopilar de forma organizada la información sobre los eventos de derrames de hidrocarburos que tuvieron impacto en la zona. Por medio de artículos de prensa (El Colombiano, Caracol Radio, EPM, El Tiempo) y páginas web (Corantioquia, EPM), se pudo evidenciar que los derrames se vienen presentando desde el año 2002 con un periodo de tiempo de 8 años sin actividad de derrames, los cuales se volvieron a presentar en el año 2011 con otro periodo de tiempo de 6 años sin actividad de derrames hasta el 2019.

Las razones por las cuales se tienen estos lapsos de tiempo son totalmente desconocidas ya que en ninguna base de datos se respalda por qué se da este suceso. Las noticias encontradas cada año fueron tomadas de fuentes confiables, ya que provienen de periódicos y páginas web certificadas y ninguna fue reportada o denunciada por publicar noticias falsas.

\begin{tabular}{rcc} 
Tipo de Evento & Fecha de Ocurrencia & Fuente de Información \\
\hline $\begin{array}{r}\text { Ecopetrol mantiene suspendido el bombeo } \\
\text { de combustibles por el Oleoducto Medellín- }\end{array}$ & 29/07/2002 & (Velásquez, 2002) \\
$\begin{array}{c}\text { Cartago, debido a una fuga de carburantes } \\
\text { detectada por rotura de manos criminales. }\end{array}$ & & \\
\hline $\begin{array}{r}\text { El estallido del poliducto Sebastopol- } \\
\text { Medellín-Yumbo, ocurrido hacia las 5 de } \\
\text { la mañana en el kilómetro 13, cuando } \\
\text { el poliducto transportaba combustible, } \\
\text { causado por hurto. }\end{array}$ & $01 / 11 / 2002$ & \\
\hline $\begin{array}{r}\text { Técnicos de Ecopetrol evalúan en } \\
\text { la mañana de este viernes el daño }\end{array}$ & $15 / 04 / 2011$ & (El Colombiano, 2011) \\
por un derrame de combustible en el & & \\
corregimiento San Cristóbal de Medellín. & & (Minuto30, 2011) \\
\hline $\begin{array}{r}\text { Deslizamiento de tierra rompió el } \\
\text { poliducto de Ecopetrol que pasa por } \\
\text { el Corregimiento de San Cristóbal en } \\
\text { Medellín. }\end{array}$ & $15 / 04 / 2011$ & \\
\hline
\end{tabular}

(Continúa en la siguiente página) 


\begin{tabular}{|c|c|c|}
\hline Tipo de Evento & Fecha de Ocurrencia & Fuente de Información \\
\hline $\begin{array}{l}\text { Corregimiento se agrava con la } \\
\text { implementación de megaproyectos viales } \\
\text { como: El primer túnel de occidente. }\end{array}$ & $21 / 01 / 2018$ & (Kavilando Radio, 2018) \\
\hline $\begin{array}{r}\text { Preocupados se encuentran los habitantes } \\
\text { de la vereda Naranjal, del corregimiento } \\
\text { de San Cristóbal, debido a las obras de } \\
\text { continuidad del Túnel de Occidente, pues } \\
\text { la primera fase de construcción afectó sus } \\
\text { viviendas y cultivos. }\end{array}$ & $28 / 01 / 2018$ & (Canal TeleMedellín, 2018) \\
\hline \multicolumn{3}{|l|}{$\begin{array}{l}\text { EPM informó que detectó contaminación } \\
\text { en las quebradas abastecedoras de la } \\
\text { planta de potabilización San Cristóbal. }\end{array}$} \\
\hline $\begin{array}{l}\text { La contaminación obliga a la empresa } \\
\text { a interrumpir el servicio de agua en los } \\
\text { sectores del centro-occidente en Medellín. }\end{array}$ & $15 / 07 / 2019$ & (Publimetro, 2019) \\
\hline $\begin{array}{r}\text { EPM detectó nuevamente contaminación } \\
\text { relacionada con hidrocarburos en las } \\
\text { quebradas abastecedoras de la planta } \\
\text { de potabilización San Cristóbal, que } \\
\text { suministra agua potable al corregimiento } \\
\text { de San Cristóbal y sectores de los barrios: } \\
\text { Antonio Nariño, Juan XXIII, Peñitas y La } \\
\text { Loma. (15 de julio) }\end{array}$ & $31 / 07 / 2019$ & $\begin{array}{l}\text { (Empresas Públicas de } \\
\text { Medellín, 2019) }\end{array}$ \\
\hline $\begin{array}{l}\text { EPM comenzó a restablecer el servicio } \\
\text { de acueducto, por un derramamiento de } \\
\text { hidrocarburos. Para garantizar el recurso } \\
\text { hídrico a la comunidad afectada, la entidad } \\
\text { reveló que con carrotanques suministró } \\
90.000 \text { litros mientras duró la emergencia. }\end{array}$ & 01/08/2019 & $\begin{array}{c}\text { (Zambrano Benavides, } \\
\text { 2019) }\end{array}$ \\
\hline $\begin{array}{r}\text { Por tercera vez en menos de un mes } \\
\text { EPM debió suspender el servicio de } \\
\text { agua, debido a que se registró una nueva } \\
\text { contaminación con hidrocarburos en las } \\
\text { quebradas que abastecen la planta de } \\
\text { potabilización de la zona. }\end{array}$ & 04/08/2019 & $\begin{array}{c}\text { (Redacción BLU Radio, } \\
\text { 2019) }\end{array}$ \\
\hline $\begin{array}{r}\text { Corantioquia ha realizado visitas de control } \\
\text { y seguimiento al territorio para determinar } \\
\text { la procedencia de la contaminación. } \\
\text { Además, un equipo técnico continuará } \\
\text { haciendo recorridos a lo largo de la } \\
\text { corriente para identificar los puntos de } \\
\text { posibles descargas, con el fin de tomar las } \\
\text { medidas que correspondan. }\end{array}$ & 06/08/2019 & (Minuto30, 2019) \\
\hline $\begin{array}{r}\text { El primer indicador que tiene Empresas } \\
\text { Públicas de Medellín, para establecer la } \\
\text { contaminación con hidrocarburos es el olor } \\
\text { del agua. }\end{array}$ & 06/08/2019 & $\begin{array}{l}\text { (Canal Regional } \\
\text { TeleAntioquia, 2019) }\end{array}$ \\
\hline $\begin{array}{r}\text { La directora de la Entidad, Ana Ligia Mora, } \\
\text { dijo que se logró determinar que parte } \\
\text { de la contaminación en el corregimiento } \\
\text { de San Cristóbal, se dio porque la } \\
\text { Concesionaria Desarrollo Vial al Mar } \\
\text {-Devimar- venía almacenando un material } \\
\text { de excavación sin tomar acciones que } \\
\text { impidieron el vertimiento de estos sobre las } \\
\text { fuentes hídricas. }\end{array}$ & 07/08/2019 & (Sinergia Informativa, 2019) \\
\hline
\end{tabular}


En la línea del tiempo (ver Figura 1) se presentan cronológicamente eventos clave, que permiten relacionar tanto los sucesos del pasado como los del presente, su descripción y comprensión. Allí se muestran los hechos cronológicamente que afectaron al Corregimiento de San Cristóbal a causa de la contaminación de fuentes hídricas por hidrocarburos.

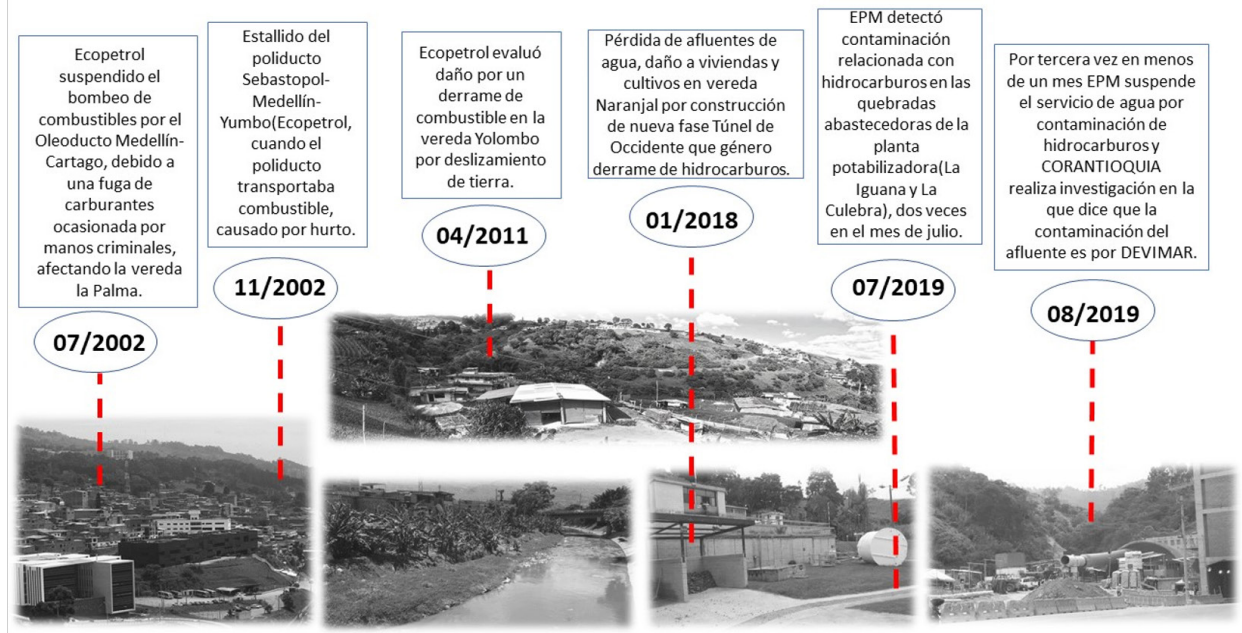

CONTAMINACION DE FUENTES HIDRICAS POR HIDROCARBUROS EN SAN CRISTOBAL A TRAVES DEL TIEMPO

Figura 1. Línea del tiempo de la ocurrencia de derrames en Corregimiento de San Cristóbal Fuente: Autoras, 2022

A partir de la elaboración de la matriz de hechos históricos y la línea del tiempo se pudo realizar un mapa de las zonas afectadas (ver Figura 2). De este mapa se puede analizar que los primeros eventos ocurridos en el año 2002 de contaminación de agua por hidrocarburos son dispersos, y afectaron tanto la centralidad como otras veredas del sector, lo que induce que la contaminación no es generada de manera focalizada, ocurre en diferentes lugares y por circunstancias diversas, como se puede ver en el mapa se encuentran localizados en las veredas del corregimiento La Palma y Yolombo. La causa de estos derrames se debe a deslizamientos de tierra o hurtos en el poliducto.

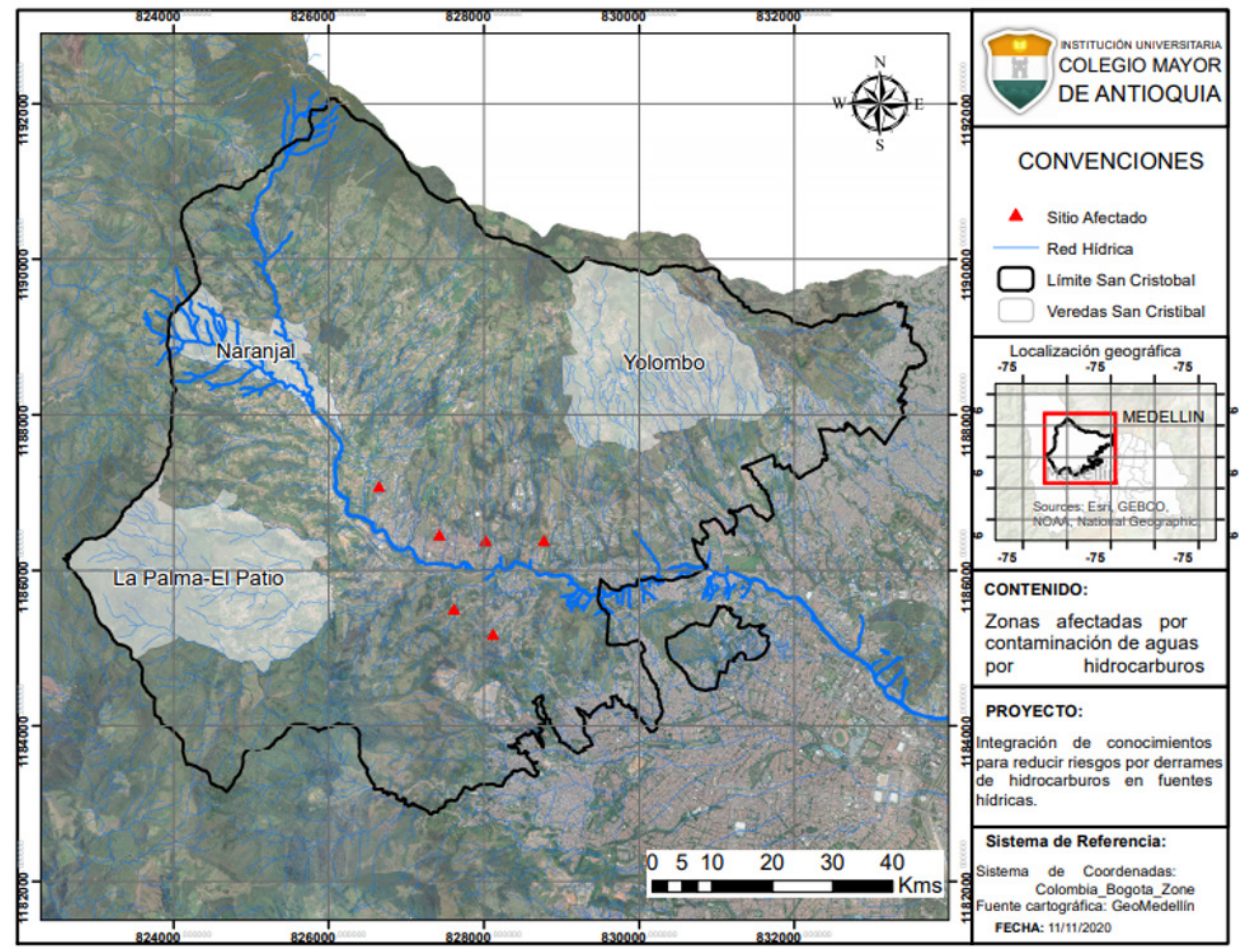

Figura 2. Mapa de zonas afectadas por contaminación de aguas por hidrocarburos Fuente: Autoras, 2022, en ArcGIS 
Por otro lado, en el mapa se aprecia cómo se pueden afectar las quebradas La Iguaná y La Culebra y la centralidad del Corregimiento de San Cristóbal por el derrame de hidrocarburos en la construcción de la segunda etapa del túnel de Occidente, hecho que necesitó una investigación por Corantioquia al consorcio Devimar, afectado principalmente en el año 2019.

Se realizó un mapa conceptual (ver Figura 3) con el fin de identificar las fuentes que generan amenaza a la prestación del servicio de agua potable en la zona por motivo de derrames, explosiones del oleoducto, acciones delictivas, teniendo como actores principales EPM, Corantioquia, Devimar y ECOPETROL. De este mapa se puede analizar cuál es la función de los diferentes actores dentro del corregimiento de San Cristóbal y cómo estuvieron involucrados en la problemática presentada.

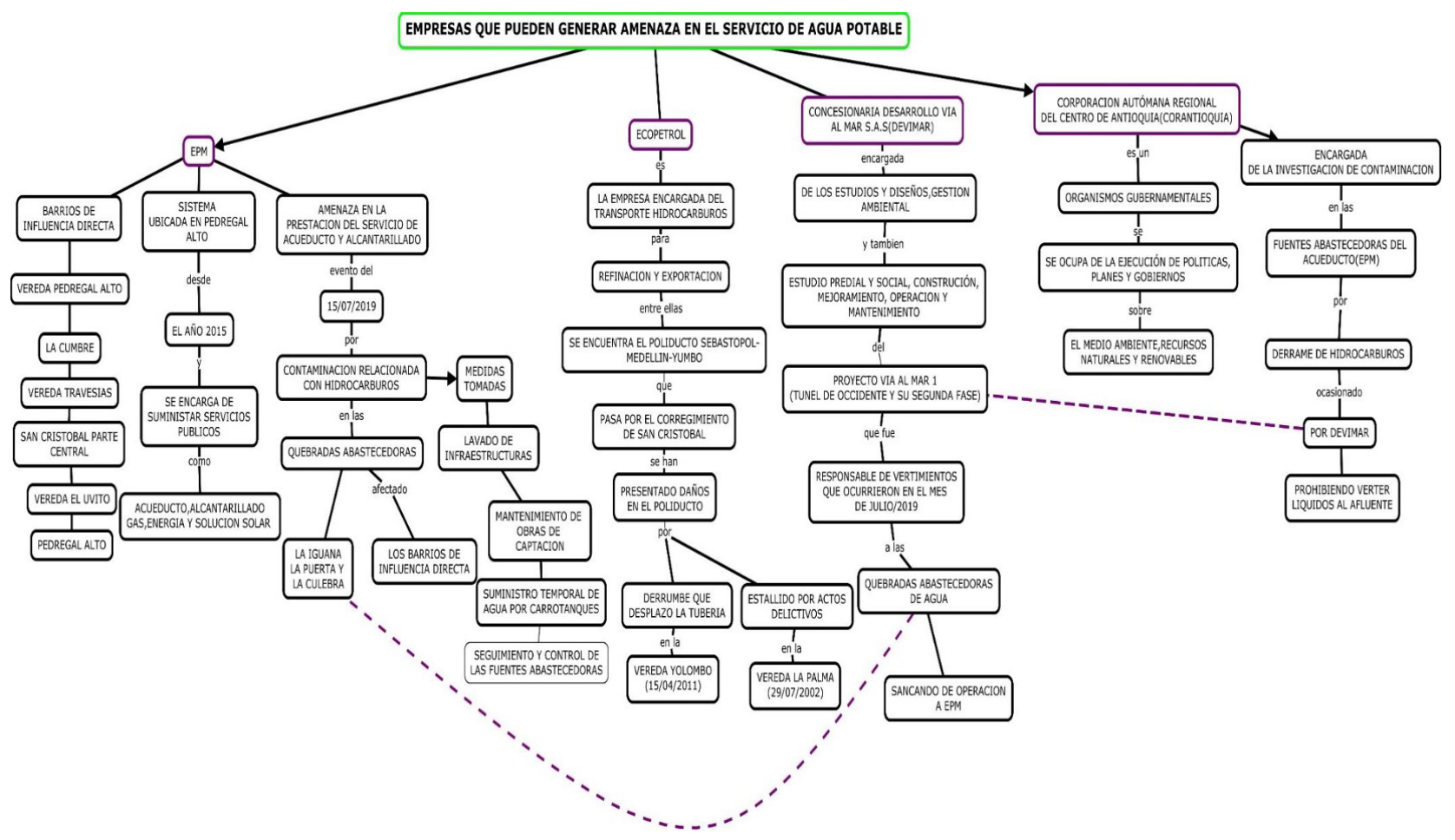

Figura 3. Mapa Conceptual de las Fuentes que Generan Amenaza Fuente: Autoras, 2022, en CmapTools

\section{Mapa de actores}

Se realizó un mapa de actores mediante un enfoque global que permitió abarcar la mayor cantidad de actores, sus relaciones y la incorporación de factores sociales, económicos y ambientales.

\section{El mapa de actores se categorizó por 4 niveles:}

Primer nivel: se encuentran los actores políticos - administrativos regionales, destacando CORANTIOQUIA y el Concejo Municipal de Gestión del Riesgo, los cuales son actores activos en el corregimiento y tomaron acción frente a la problemática.

Segundo nivel: están ubicados los actores encargados de la protección y control, siendo los responsables de la atención a emergencia en los eventos históricos de derramamiento de hidrocarburos en las fuentes hídricas.

Tercer nivel: se encuentran aquellas entidades Político-Administrativas sectoriales, encargadas de la planificación estratégica, comunicación del riesgo y la protección financiera de la comunidad.

Cuarto nivel: están los actores económicos, con 3 principales organizaciones (EPM, ECOPETROL y DEVIMAR), las cuales cuentan con infraestructura en el corregimiento de San Cristóbal, prestando un servicio a la comunidad y tienen la obligación de la preservación de los recursos naturales del sector. En este nivel también se destacan los campesinos los cuales aportan a la economía del corregimiento y son los más vulnerables frente a la contaminación de las fuentes hídricas. 
En este mapa de actores (ver Figura 4) se puede identificar el flujo de información, cooperación y potenciales conflictos mediante la relación hecha entre los diferentes actores de cada nivel. Se observa que existe una coordinación que permite que cada uno de estos actores se conecten de manera constante debido al intercambio de información que hay entre ellos; a su vez se puede identificar la cooperación que hay buscando beneficio para el desarrollo de Corregimiento, siempre y cuando se tengan en cuenta los potenciales conflictos que hay en la localidad para poder dar solución a estos. El mapa abarca un contexto global el cual nos permite tener una visión amplia y clara de la problemática para saber qué acciones tomar.

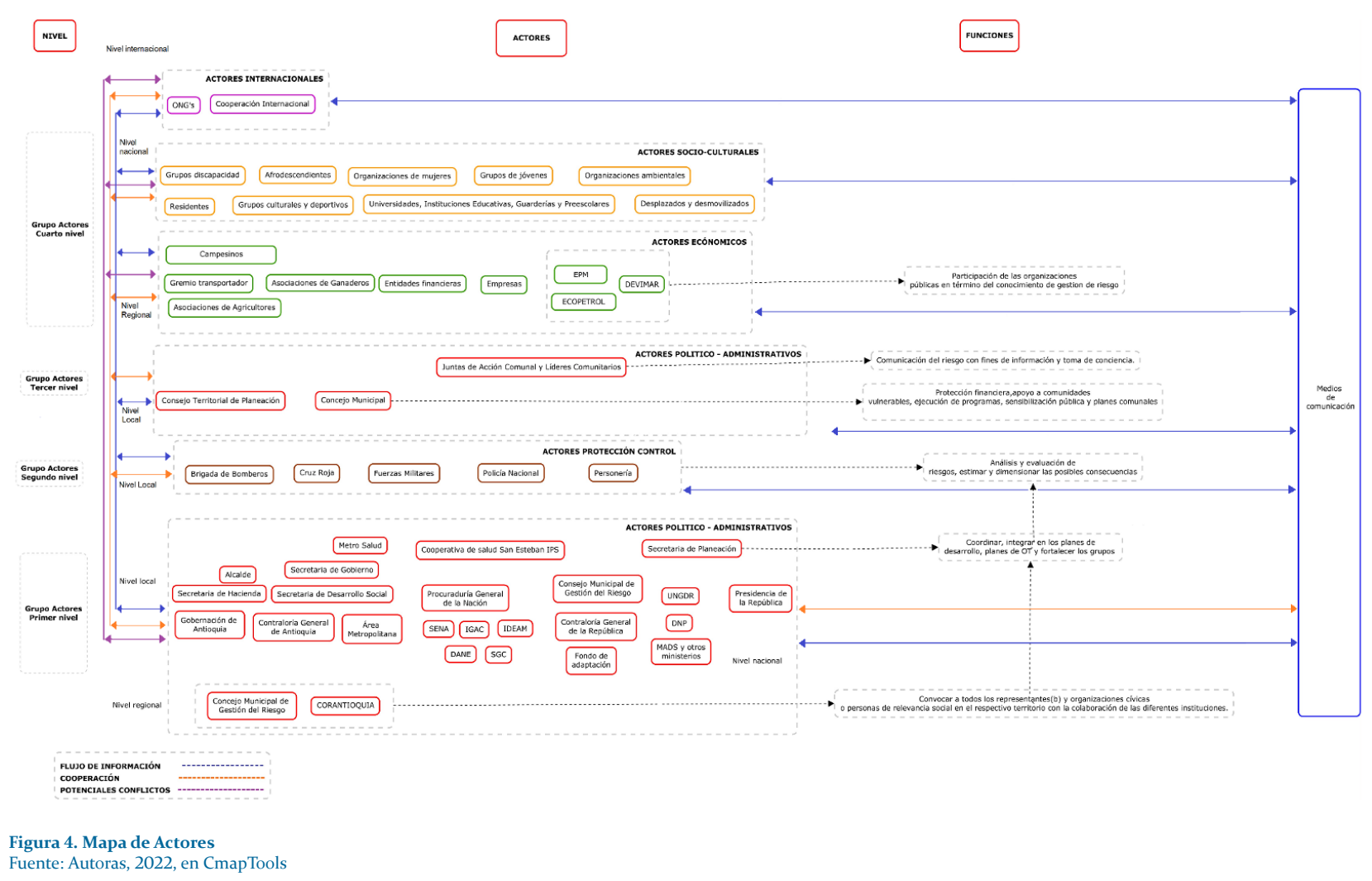

\section{Cartografiado de afectaciones}

Para la creación de la cartografía fue necesario la participación de la comunidad del corregimiento de San Cristóbal para recolección de datos sobre el grado de afectación de la comunidad por la contaminación de hidrocarburos en el agua.

Primero, se creó una página web ${ }^{1}$ por medio de la aplicación Wix, además se incorporó en la página una infografía con información más concisa que incluyera el enlace para acceder a la encuesta.

La Encuesta a la comunidad del Corregimiento consistió en una integración de conocimiento local como medida de reducción de riesgo por derrame de hidrocarburos en fuentes hídricas del corregimiento de San Cristóbal. En los Anexos se presentan los elementos que la componen.

\section{Resultados de la encuesta dirigida a la comunidad}

Los resultados arrojados por las encuestas dan un total de 38 respuestas, de las cuales 37 fueron válidas y 1 inválida ya que esta persona no reside dentro del corregimiento. Se ha obtenido una muestra representativa para delimitar la zona.

En promedio la población convive en un núcleo familiar entre 3 y 4 personas por vivienda y que la mayoría de los integrantes de estos núcleos son adultos, el mayor porcentaje $(42,1 \%)$ de asentamiento poblacional está en la parte central del corregimiento y el otro porcentaje menor en las veredas Travesías, el Llano, la Palma, Pedregal Alto y la Loma. Por otro lado, los resultados de la encuesta arrojaron que las personas que se vieron más afectadas por la suspensión de cortes de servicio de acueducto fueron los usuarios del servicio de EPM. En la encuesta se observó que más del $50 \%$ de la comunidad está mal informada, ya que no saben cuál es el verdadero motivo 
por el cual se les suspendió el servicio. Solo el $36.9 \%$ de la comunidad es la que se encuentra bien informada acerca de la suspensión del servicio, presentando algún síntoma relacionado con la ingesta de agua potable contaminada por hidrocarburos en sus hogares.

Se evidenció que los entes del corregimiento presentan fallas en la forma de comunicar y dar a conocer sus estrategias (participación ciudadana, educación ambiental y conocimientos de gestión del riesgo), ya que un $63.2 \%$ de la población desconoce totalmente de la existencia de estas. Asimismo, el $86.8 \%$ de la población presenta interés en participar en proyectos de educación, sensibilización y capacitaciones en pro de mejorar todos sus conocimientos socioambientales y así poder mitigar los impactos negativos que genera la contaminación de fuentes hídricas por hidrocarburos.

Gracias a los resultados y análisis de la encuesta se pudo plasmar en la cartografía (ver Figura 5) la ubicación de las personas afectadas mediante el grado de afectación de la comunidad frente a la problemática expuesta; la cual se clasificó por la metodología diamante: siendo rojo(inminente), naranja (probable), verde (posible).

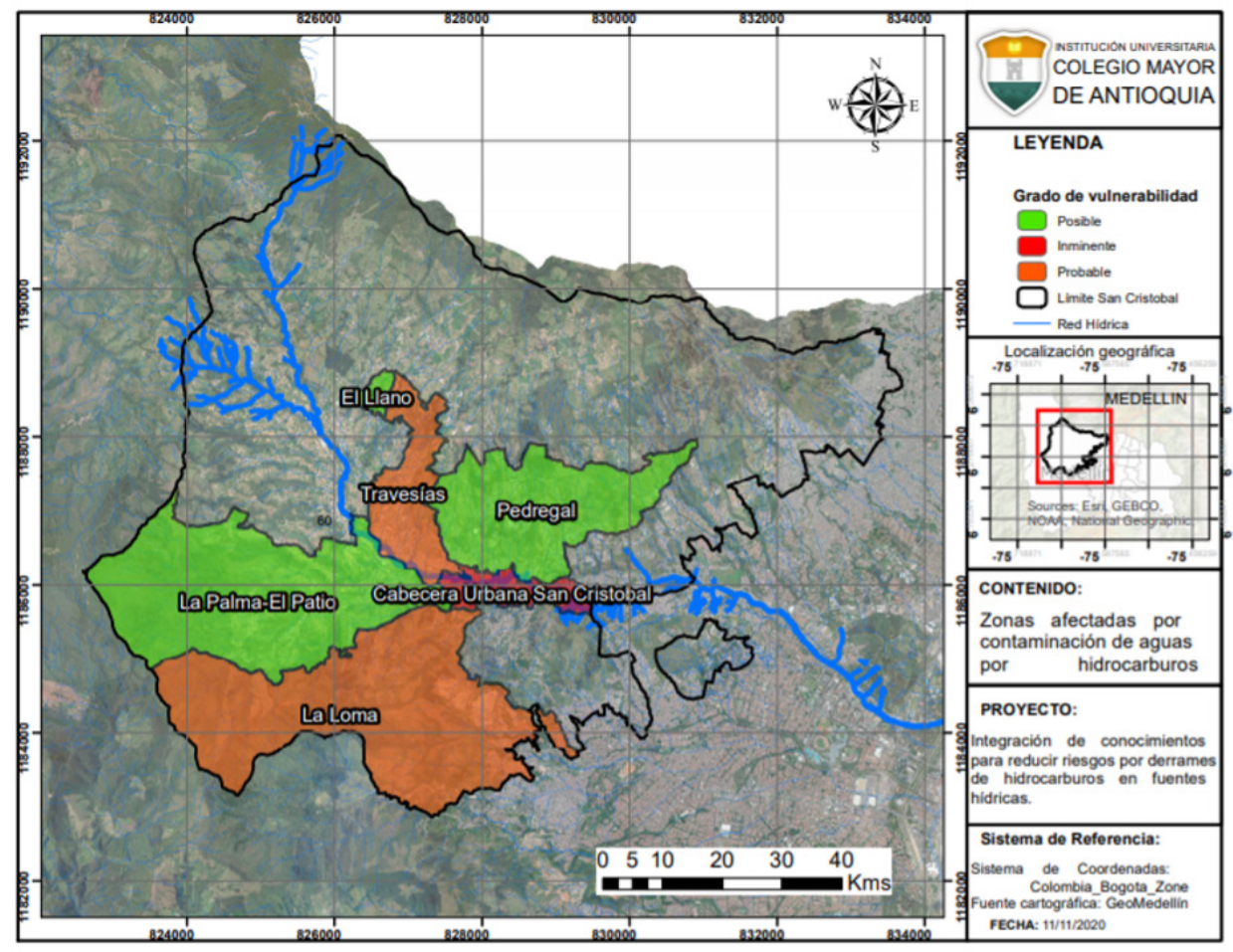

Figura 5. Cartografía del sector donde está ubicada la comunidad vulnerable por la contaminación de hidrocarburos en fuentes hídricas

Fuente: Autoras, 2022, en ArcGIS

\section{Formulación de estrategias}

Se recolectó información a través de la Junta de Acción Local del Corregimiento de San Cristóbal sobre planes de reducción del riesgo que poseen, a través de una encuesta presencial en sus instalaciones ubicadas en la centralidad del Corregimiento (Tabla 2).

\begin{tabular}{ll}
$\begin{array}{l}\text { Encuesta dirigida a la Junta de Acción Local } \\
\text { (JAL): }\end{array}$ & $\begin{array}{l}\text { Resultados de la entrevista dirigida a la Junta } \\
\text { de Acción Local (JAL): }\end{array}$ \\
\hline $\begin{array}{l}\text { ¿Cuáles son las Acciones principales para mitigar } \\
\text { cuando ocurre una amenaza por contaminación por }\end{array}$ & $\begin{array}{l}\text { No poseen plan de contingencia, ya que dijeron } \\
\text { qudrocarburos? }\end{array}$ \\
\hline $\begin{array}{ll}\text { ¿Cuál es la estructura organizacional para llevar a } \\
\text { cabo los planes de contingencia? }\end{array}$ & $\begin{array}{l}\text { No poseen estructuración organizacional, ya } \\
\text { que lo que ellos hacen únicamente es notificar la } \\
\text { ocurrencia de los incidentes. }\end{array}$ \\
\hline
\end{tabular}

(Continúa en la siguiente página) 
(Continuación)

\begin{tabular}{ll}
$\begin{array}{ll}\text { Encuesta dirigida a la Junta de Acción Local } \\
\text { (JAL): }\end{array}$ & $\begin{array}{l}\text { Resultados de la entrevista dirigida a la Junta } \\
\text { de Acción Local (JAL): }\end{array}$ \\
\hline $\begin{array}{l}\text { ¿Cómo manejan la información y de qué manera } \\
\text { preparan los comunicados dirigidos hacia la } \\
\text { comunidad y las instituciones cuando ocurre una } \\
\text { emergencia? }\end{array}$ & $\begin{array}{l}\text { La Junta de Acción Local sugirió que este proceso } \\
\text { no lo hacen, debido a que ellos únicamente } \\
\text { notifican la ocurrencia de una emergencia a la línea } \\
\text { telefónica de emergencias 123. }\end{array}$ \\
\hline $\begin{array}{l}\text { ¿De qué manera se da a conocer el riesgo de } \\
\text { hidrocarburos en cuerpos hídricos a la comunidad } \\
\text { cuando ocurre un evento de contaminación? }\end{array}$ & $\begin{array}{l}\text { No realizan, por temor a represalias de entes o } \\
\text { personas malintencionadas. }\end{array}$ \\
\hline $\begin{array}{l}\text { ¿De qué manera se actúa cuando ocurre una } \\
\text { contingencia de contaminación por hidrocarburos? }\end{array}$ & Se comunican o notifican el incidente. \\
\hline $\begin{array}{l}\text { ¿El corregimiento cuenta con protocolos de } \\
\text { emergencia cuando ocurren emergencias? }\end{array}$ & No cuenta con un protocolo establecido. \\
\hline $\begin{array}{l}\text { ¿Existen en el corregimiento organismos de } \\
\text { socorro como la Defensa Civil Colombiana, la Cruz } \\
\text { Roja? }\end{array}$ & \begin{tabular}{l} 
Solo se cuenta con la existencia de la Defensa \\
\hline
\end{tabular}
\end{tabular}

Tabla 2. Encuesta dirigida a la Junta de Acción Local

Fuente: Autoras, 2022

Según las respuestas de la JAL, no poseen ningún vínculo con este tipo de eventos de gestión del riesgo, en el corregimiento no se cuenta con planes de gestión del riesgo que ayuden a la reducción de vulnerabilidad y reiteran que son necesarios para el corregimiento en caso de cualquier emergencia, estar preparados y actuar de forma correcta.

Para la implementación de las estrategias de educación y participación, se realizó una capacitación virtual con la comunidad de San Cristóbal por la plataforma de Google Meet para videoconferencias, la cual se llevó a cabo con la participación de 24 personas. En la capacitación se dio a conocer la temática del proyecto, se realizó una contextualización de los hechos históricos de eventos de contaminación por hidrocarburos en las fuentes hídricas del corregimiento y se presentaron las evidencias por medio de noticias de diferentes años hasta llegar al evento más reciente ocurrido en el 2019.

Luego se pasó al cuerpo de la capacitación donde se explicó cómo identificar cuando el agua del grifo está contaminada por hidrocarburos, por sus características (olor, sabor, color), afectaciones a la salud y cómo actuar en caso de que ingiera agua con hidrocarburos, todo esto encaminado hacia una gestión del riesgo oportuna para la comunidad afectada, para la oportuna acción ante la ocurrencia del derrame y que se presente la posibilidad que la comunidad tenga contacto con fuentes hídricas contaminadas con hidrocarburos. Para ello es importante las medidas de prevención y de corrección tales como; el autocuidado, el reconocimiento del agua contaminada con hidrocarburos, síntomas, entidades a las cuales deben acudir en caso tal de intoxicación, cuáles son los centros asistenciales de salud más cercanos según su sector de residencia y las propuestas para la comunidad.

Al final de la capacitación se propuso a la comunidad la creación de grupos de gestión del riesgo ya que con esto podemos saber qué amenaza al corregimiento, también es importante saber cómo manejar y controlar el riesgo, ya que con estas medidas se pretende reducir el impacto tanto al ambiente, como a la economía y a la sociedad. Los participantes se expresaron interesados y entusiasmados con la idea de implementar las medidas de reducción de la vulnerabilidad (red de alerta comunitaria y conformación del comité para la gestión del riesgo de desastres), se mostraron dispuestos a ser parte de estas iniciativas para el corregimiento y dieron opiniones como:

» "Me parece genial la propuesta, así podríamos estar más pendientes del corregimiento y podríamos reducir futuros riesgos".

» "La comunidad es la primera línea de respuesta a un incidente y sobre todo los más afectados, ellos deben estar apoderados de la gestión del riesgo en el conocimiento y ser parte fundamental en la reducción".

» "Si esto es muy necesario e importante que las Juntas de Acción Comunitarias de cada barrio y vereda estén capacitadas de esto". 
» "Las empresas del sector deberían de tener un plan en conjunto con el Área Metropolitana y/o Corantioquia para mitigar este tipo de contaminación".

" "Ecopetrol históricamente ha mantenido un trabajo muy cercano con los equipos de respuesta en dotación y capacitación, pero eso es tan importante como lo que ustedes proponen. Se debería fortalecer su propuesta y darle viabilidad".

Con estos comentarios se puede decir que se ve viable un fortalecimiento de la gestión del riesgo en el corregimiento de San Cristóbal, haciendo partícipes a la comunidad ya que hay un interés por parte de ellos, además es de gran importancia para que ellos tengan un reconocimiento de su territorio y se fortalezca su sentido de pertenencia y compromiso por el mismo.

\section{CONCLUSIONES Y RECOMENDACIONES}

En el periodo 2002 - 2019 se han presentado derrames por hidrocarburos en la comunidad y con la ayuda de este panorama se pudo determinar el área de estudio por medio de una línea de tiempo, en donde se obtuvo información que se enlaza con el paso de los años y su relación por el mayor número de impactos, ya sea por la afectación a la infraestructura del sector.

El mapa de actores es un indicativo que muestra las interrelaciones que existen entre los diferentes actores, mediante un trabajo articulado, que permite un flujo de información entre ellos. Se recopiló la mayor cantidad de información sobre las condiciones de riesgos asociadas a la contaminación de hidrocarburos, y sus mayores afectaciones a la comunidad y al ambiente del Corregimiento de San Cristóbal, complementando con la realización de la cartografía construida con la participación de la comunidad a través de una encuesta, la cual nos permitió sacar el grado de afectación y poder ver el riesgo que genera en la comunidad la problemática planteada, reflejado en la cabecera urbana y en las veredas de Travesías y Pedregal alto.

Se entiende que la comunidad que reside en el Corregimiento y los ecosistemas allí localizados, son vulnerables ante la contaminación de fuentes hídricas por hidrocarburos ya que cada evento ocurrido lo posiciona como un sitio expuesto, susceptible a este tipo de amenazas. Por consiguiente, tiene problemas de vigilancia y asesoramiento a la comunidad aledaña al poliducto; así mismo, debe haber una armonía en lo que se plantea en el plan de desarrollo local, ya que, en materia de gestión del riesgo, esta es una directriz para reducir la vulnerabilidad del sector en cuanto a desastres, por lo tanto, se evidencia que la alineación que hay referente al contexto de los eventos de contaminación y el plan de desarrollo local es muy débil.

Es importante la creación de grupos de gestión del riesgo con la comunidad interesada del Corregimiento para que estos puedan tomar medidas de acción participativa, realizar propuestas orientadas al mejoramiento de las condiciones de su territorio. Esta iniciativa se propuso mediante la capacitación realizada a la comunidad, donde todos se mostraron muy motivados en ser partícipes de la gestión del riesgo del corregimiento, para que cuando se vuelva a presentar un derrame de hidrocarburos en sus fuentes hídricas se dé una acción oportuna y no como en ocasiones anteriores donde la centralidad y veredas cercanas a ella se vieron afectadas y no tenían conocimiento de cómo actuar.

Por último, es importante implementar capacitaciones con la comunidad por parte de los actores implicados, con el fin de fortalecer la comunicación, aumentar la preparación en casos de desastre y así poder tener una mejor capacidad de respuesta por una emergencia.

La incorporación de instituciones para la gestión del riesgo de desastres y el fortalecimiento de estas, para que por medio de intervenciones prospectivas y correctivas reduzcan la vulnerabilidad presente; también se recomienda involucrar los diferentes medios de comunicación del sector para implementar estrategias de alerta y una buena actuación de la comunidad y de los diferentes entes que operan en el sector.

Se propone incluir proyectos relacionados con la gestión del riesgo de desastres por hidrocarburos en el plan de desarrollo local para reducir la vulnerabilidad. Y finalmente, se propone orientar en la comunidad para que se promueva la red de alerta comunitaria del corregimiento, así como también el comité para la gestión del riesgo de desastres, para que de esta manera se fortalezca la seguridad en el sector.

A continuación, se resumen alternativas para el corto, mediano y largo plazo (ver Tabla 3 y Tabla 4). 


\begin{tabular}{|c|c|c|}
\hline Corto & Mediano & Largo \\
\hline \multicolumn{3}{|l|}{$\begin{array}{l}\text { Conocimiento del riesgo por } \\
\text { medio de una categorización por } \\
\text { eventos de desastres histórico }\end{array}$} \\
\hline \multicolumn{3}{|l|}{ Análisis de fuentes de amenaza } \\
\hline \multicolumn{3}{|l|}{$\begin{array}{l}\text { Identificación del flujo de } \\
\text { información, cooperación y } \\
\text { potenciales conflictos entre los } \\
\text { actores }\end{array}$} \\
\hline \multicolumn{3}{|c|}{ Participación Comunitaria } \\
\hline \multicolumn{3}{|c|}{ Educación ambiental } \\
\hline \multicolumn{3}{|c|}{ Mejoramiento de relaciones sociales } \\
\hline \multirow[t]{2}{*}{$\begin{array}{l}\text { Planificación de zonas afectadas } \\
\text { por contaminación de aguas por } \\
\text { hidrocarburos }\end{array}$} & & \\
\hline & $\begin{array}{l}\text { Procesos sancionatorios por el } \\
\text { hurto de hidrocarburos }\end{array}$ & \\
\hline \multicolumn{3}{|l|}{$\begin{array}{l}\text { Licenciamiento ambiental para } \\
\text { proyectos en la zona }\end{array}$} \\
\hline \multicolumn{3}{|c|}{ Promoción y gestión de la norma para la gestión del riesgo desastres (Ley 1523-2012) } \\
\hline & $\begin{array}{l}\text { Fortalecimiento de la capacidad } \\
\text { de respuesta de la comunidad de } \\
\text { San Cristóbal }\end{array}$ & \\
\hline $\begin{array}{l}\text { Control de enfermedades a } \\
\text { causa contaminación de fuentes } \\
\text { hídricas por hidrocarburos }\end{array}$ & & \\
\hline
\end{tabular}

Tabla 3. Intervención Prospectiva (Prevención del riesgo) Fuente: Autoras, 2022

\begin{tabular}{l|l|}
\hline Corto & Mediano \\
\hline $\begin{array}{l}\text { Implementación de un sistema de alerta temprana } \\
\text { la comformación de una brigada de emergencia para }\end{array}$ & \\
\hline Recuperación de las fuentes hídricas contaminadas \\
\hline Recuperación del suelo \\
\hline Seguimiento y control del agua en las fuentes de abastecimiento \\
\hline Ejecución de proyectos de gestión del riesgo \\
\hline
\end{tabular}

Tabla 4. Intervención correctiva (mitigación del riesgo)

Fuente: Autoras, 2022

\section{REFERENCIAS}

Alcaldía de Medellín. (2015). Plan de desarrollo local. Corregimiento San Cristóbal. Alcaldía de Medellín. https://www.medellin.gov.co/irj/go/km/docs/pccdesign/SubportaldelCiudadano_2/ PlandeDesarrollo_o_17/ProgramasyProyectos/Shared Content/Documentos/2015/Planes de desarrollo Local/COMUNA 6o San Cristobal.pdf

Amaya Navas, O.D. (2012). El desarrollo sostenible y el derecho fundamental a gozar de un ambiente sano. Universidad Externado de Colombia.

Asamblea General de Las Naciones Unidas. (2015). Marco de Sendai para la Reducción del Riesgo de Desastres 2015-2030. ONU.

Benavides López De Mesa, J., Quintero, G., Guevara Vizcaíno, A.L., Carolina, D., Cáceres, J., Milena Gutiérrez Riaño, S., \& García, J.M. (2005). Bioremediación de suelos contaminados con hidrocarburos derivados del petróleo. Nova, 4(5), 82-90. 
Canal Regional TeleAntioquia. (2019, 6 de agosto). Preocupación por contaminación de agua en San Cristóbal. Canal Regional TeleAntioquia https://www.teleantioquia.co/featured/ preocupacion-por-contaminacion-de-agua-en-san-cristobal/

Canal TeleMedellín. (2018, 28 de enero). Preocupación en San Cristóbal por obras de continuidad del Túnel de Occidente. Canal TeleMedellín. https://telemedellin.tv/ san-cristobal-tunel-de-occidente/241138/

Caracol Radio Medellín. (2019, 3 de agosto). Devimar habría contaminado el acueducto de San Cristóbal. Caracol Radio Medellín. https://caracol.com.co/emisora/2019/o8/o3/ medellin/1564861940_341263.html

Congreso de la República de Colombia. (2012). Ley 1523 de 2012. Por la cual se adopta la Política Nacional de Gestión del Riesgo de Desastres y se dictan otras disposiciones. Congreso de la República de Colombia.

Cuartas Nústez, D.C., Paredes Cuervo, D., \& Cubillos Vargas, J. (2014). Bioremediation for degradation of total hydrocarbons present in the sediments of a fuel service station. Revista Técnica de La Facultad de Ingeniería Universidad Del Zulia, 37(1), 20-28.

El Colombiano. (2011, 15 de abril). Ecopetrol evalúa daño en poliducto en el corregimiento de San Cristóbal. El Colombiano. https://www.elcolombiano.com/historico/dano_en_poliducto_en_el_ corregimiento_de_san_cristobal_es_evaluado_por_ecopetrol-KGEC_129762.

Empresas Públicas de Medellín (EPM). (2019). EPM interrumpió el servicio de acueducto en San Cristóbal y zonas aledañas, debido a contaminación de quebradas por un tercero. EPM. https://www. epm.com.co/site/epm-interrumpio-el-servicio-de-acueducto-en-san-cristobal-y-zonas-aledanas

Galeano Balvuena, M.F. (2015). Implicaciones de un modelo para la gestión del riesgo de desastres: Caso Comunidad Andina. Universidad Militar Nueva Granada.

Gil Castrillón, Â.M. (2018). Diagnóstico comparativo en la adopción de las directrices para elaboración del Plan de Gestión del Riesgo en 4 acueductos rurales del Municipio de Envigado, en el marco del Decreto 2157 de 2017 . (Postgrado). Institución Universitaria Colegio Mayor de Antioquia.

Gudynas, E. (2003). Ecología, economía y ética del desarrollo sostenible (4a edición). ILDIS FES (Instituto Latinoamericano de Investigaciones Sociales) \& Ediciones Abya Yala.

Herrera, J.C. (2002, 1 de noviembre). Tragedia en poliducto. El Tiempo. https://www.eltiempo.com/ archivo/documento/MAM-1335988

Kavilando Radio. (2018, 21 de enero). Campesinos y campesinas de Medellín, protestan en la autopista a occidente y se declaran "en vía de extinción. Kavilando Radio https://kavilando.org/lineas-kavilando/ formacion-genero-y-luchas-populares/5983-campesinos-y-campesinas-de-medellin-protestan-enla-autopista-a-occidente-y-se-declaran-en-via-de-extincion

Williams, D.S., Costa, M.M., Celliers, L., \& Sutherland, C. (2018). Informal settlements and flooding: Identifying strengths andweaknesses in local governance for water management. Water (Switzerland), 10(7), 871. https://doi.org/10.3390/w10070871

Mesa de Concertación para la Lucha contra la Pobreza, \& Cáritas del Perú. (2009). Gestión del riesgo de desastres para la planificación del desarrollo local. Mesa de Concertación. www.mesadeconcertacion. org.pe

Minutozo. (2011, 15 de abril). Emergencia Ecológica En San Cristóbal Por Rompimiento de Poliducto de Ecopetrol. Minutozo. https://www.minutozo.com/ emergencia-ecolgica-en-san-cristbal-por-rompimiento-de-poliducto-de-ecopetrol/41141/

Minutozo. (2019, 6 de agosto). Corantioquia investiga contaminación a las quebradas en San Cristóbal. Minutozo. https://www.minutozo.com/ corantioquia-investiga-contaminacion-a-las-quebradas-en-san-cristobal/869562/

Prieto Diaz, V., \& Martínez de Villa Pérez, A. (1999). La contaminación de las aguas por hidrocarburos: un enfoque para abordar su estudio. Revista Cubana Higiene Epidemiología, 37(1), 13-20.

Publimetro. (2019, 15 de julio). ¡Atención! Por contaminación se suspende suministro de agua en el centro - occidente de Medellín. Publimetro. https://www.publimetro.co/co/medellin/2019/o7/15/ atencion-contaminacion-se-suspende-suministro-agua-centro-occidente-medellin.html

Redacción BLU Radio. (2019, 4 de agosto). EPM restableció el servicio de agua en San Cristóbal y cuatro barrios de Medellín. BLU Radio. https://www.bluradio.com/nacion/ epm-restablecio-el-servicio-de-agua-en-san-cristobal-y-cuatro-barrios-de-medellin 
Romero Orjuela, N., Madrid Gómez, L.F. \& López, C.V. (2015). Valoración económica del impacto de la contaminación hídrica por la actividad de hidrocarburos del Campo Zoe sobre la salud humana en la Quebrada San Albertico, ubicada entre los municipios de San Martín y San Alberto en el Departamento del Cesar. (Pregrado). Universidad Distrital Francisco José De Caldas.

Rodríguez-Gaviria, E.M. (2016). Diseño metodológico para la evaluación del riesgo por inundación a nivel local con información escasa. Universidad Nacional de Colombia.

Sinergia Informativa. (2019, 7 de agosto). Devimar Contaminó Aguas de San Cristóbal: Corantioquia. Sinergia Informativa. https://www.sinergiainformativa.com.co/ devimar-contamino-aguas-de-san-cristobal-corantioquia/

Velásquez Arias, J.A. (2017). Contaminación de suelos y aguas por hidrocarburos en Colombia. Análisis de la fitorremediación como estrategia biotecnológica de recuperación. Revista de Investigación Agraria y Ambiental, 8(1), 151-167. https://doi.org/10.22490/21456453.1846

Velásquez, J.L. (2002, 29 de julio). Por acción criminal, suspendido servicio de Ecopetrol. Caracol Radio. https://caracol.com.co/radio/2002/o7/29/nacional/102789360o_033468.html

Zambrano Benavides, D. (2019, 1 de agosto). Restablecen servicio de acueducto en San Cristóbal y cuatro barrios de Medellín. El Colombiano. https://www.elcolombiano.com/antioquia/epm-restableceservicio-de-acueducto-en-medellin-y-san-cristobal-tras-superar-contaminacion-por-hidrocarburosNA11339206

\section{ANEXOS}

\section{Encuesta a la comunidad del Corregimiento}

¿Cuántas personas viven con usted?

De las personas que viven con usted cuales son:
a. Niños
b. Jóvenes
c. Adultos
d. Adultos mayores

¿Dónde está ubicada su vivienda?
a. San Cristóbal parte central
b. Vereda del Corregimiento de San Cristóbal
C. Otra

Si la respuesta anterior fue Vereda... ¿En cuál vereda vives? si no vives en alguna vereda prosigue con la encuesta y escribe NO APLICA

¿Qué empresa de acueducto le presta servicio a su hogar?
a. Acueducto veredal
b. Acueducto de EPM
C. No cuenta con el servicio de acueducto

¿Se vio afectado por la suspensión del servicio de acueducto en su hogar en el año 2019?
a. Si
b. No
c. No sabe 
De las siguientes opciones, ¿Alguna fue la causa de la suspensión del servicio de acueducto en su hogar?
a. Mantenimiento
b. Daño en tuberías
C. Contaminación especialmente por hidrocarburos
d. Ninguna de las anteriores

Si consumió agua de la llave de su hogar ¿Recuerda haber presentado alguno de los siguientes síntomas? (año de estudio 2019)
a. Diarrea
b. Dolor de estómago
c. Vómito
d. Dolor de cabeza
e. Ninguna de las anteriores

¿Conoce la existencia de estrategias en el corregimiento? tales como:
a. Gestión del riesgo de desastres
b. Educación ambiental
c. Participación ciudadana
d. Ninguna de las anteriores

¿Le gustaría formar parte alguna de las estrategias previamente mencionadas?
a. Si
b. No
c. Tal vez 